\title{
Preparation of Poly (N,N-diphenyl-4-amine) as the Cathode and Its Electrochemical Properties
}

\author{
Chang Su ${ }^{*}$, Bing Han, Yue Sun, Guosheng Wang, Lihuan Xu* \\ College of Chemical Engineering, Shenyang University of Chemical Technology, Shenyang, China \\ Email address: \\ suchang@syuct.edu.cn (Chang Su), xulihuanss@163.com (Lihuan Xu) \\ ${ }^{*}$ Corresponding author
}

To cite this article:

Chang Su, Bing Han, Yue Sun, Guosheng Wang, Lihuan Xu. Preparation of Poly (N,N-diphenyl-4-amine) as the Cathode and Its Electrochemical Properties. Science Discovery. Vol. 6, No. 4, 2018, pp. 249-256. doi: 10.11648/j.sd.20180604.14

Received: June 29, 2018; Accepted: August 1, 2018; Published: August 10, 2018

\begin{abstract}
Triphenylamine as well as its polymer have been applied in the fields of photoelectricity and energy storage. In this paper, the pyridine ring structure was introduced into the triphenylamine structure to successfully synthesize N, $\mathrm{N}$-diphenyl-4-amine monomer (DPN), and the its single crystal was then cultured. And its homopolymer (PDPN) was further prepared by chemicaloxidation method. The obtained poly (triphenylamine) derivative-PDPN was used as cathode material to study its electrochemical and battery performance. As the result, the chemical structure of the monomer was confirmed by NMR, MS and single crstal analysis. And the chemical structure of PDPN was confirmed by infrared spectroscopy and ultraviolet spectroscopy. Furthermore, the battery performance test shows that PDPN electrode has two obvious voltage platforms in the voltage range of 4.1-4.3 V and 1.5-1.8 V, in which the initial discharge specific capacity was $92.3 \mathrm{mAh} \cdot \mathrm{g}^{-1}$. After $50 \mathrm{cycles}$, its discharge specific capacity decreased from $92.3 \mathrm{mAh} \cdot \mathrm{g}^{-1}$ to $73.2 \mathrm{mAh} \cdot \mathrm{g}^{-1}$ with about $20.7 \%$ capacity loss.
\end{abstract}

Keywords: Polytriphenylamine, Lithium Battery, Organic Cathode, Electrochemical Propeerties

\section{聚（N，N-二苯基吡啶-4-胺）正极材料的制备与电化学性能研究}

苏畅”, 韩冰, 孙越, 王国胜, 徐立环 ${ }^{*}$

化学工程学院, 沈阳化工大学, 沈阳, 中国

邮箱:

suchang@syuct.edu.cn (苏畅), xulihuanss@163.com（徐立环）

摘要: 三苯胺及其聚合物在光电、储能领域具有广泛的应用前景。本文以三苯胺为研究对象, 通过在三苯胺结构中 引入吡啶环结构, 成功合成 $\mathrm{N}, \mathrm{N}$-二苯基吡啶-4-胺（DPN）化合物, 并培养出了其单晶, 然后通过化学氧化法成功 制备了其均聚物（PDPN）。采用制备的聚三苯胺衍生物PDPN为正极材料, 研究其电化学和电池性能。通过核磁氢 谱和质谱以及单晶的分析确认了单体的化学结构。通过红外光谱以及紫外光谱测试证实了三种聚合物的结构。电池 性能测试发现, PDPN电极在4.1-4.3V和1.5-1.8V电压范围内有两个明显的电压平台, 且首次放电比容量为 $92.3 \mathrm{mAh} \cdot \mathrm{g}^{-1}$ 。 经过 50 次循环后其实际容量从刚开始的 $92.3 \mathrm{mAhg}^{-1}$ 衰减到了 $73.2 \mathrm{mAhg}^{-1}$ ，大约 $20.7 \%$ 的容量损失。

关键词: 聚三苯胺, 锂电池, 有机正极, 电化学性能 


\section{1. 引言}

随着笔记本电脑、手机、数码相机和电动汽车等产品 的日渐发展, 对高比能量、高比功率和高安全性的电源提 出更加迫切的需求, 这极大地推动了二次锂离子储能电池 相关研究的不断进步。电极材料是锂离子电池技术的重要 构成部分, 而正极材料是目前制约锂离子电池发展的主要 瓶颈。相比无机正极材料, 有机或聚合物正极材料具有原 料来源丰富, 分子结构可设计, 储能密度高, 环境友好和 易于加工的柔韧性等特点, 近年来在锂电池技术研究领域 引起广泛关注并逐渐成为研究的热点。有机或聚合物正极 材料主要包括, 导电聚合物 (聚苯胺、聚噻吩、聚吡咯等) [1-4]、有机含硫化合物 [5-8]、葱醌类和含有羰基基团化合 物[9-12]和自由基聚合物等[13-16]。

三苯胺及其衍生物聚合物是导电自由基型聚合物, 由 于它们具有超快的电子传递速率和良好的空穴传输能力, 被广泛地应用于有机电致发光材料, 光导材料和有机太阳 能电池材料 [17-21]。此外, 聚三苯胺及其衍生物聚合物含 有三苯胺基础单元, 在其充放电过程中具有可逆的氧化还 原反应[22-24], 这使得探索三苯胺及其衍生物聚合物作为 超级电容器或者锂离子电池的电极材料在储能领域中的 应用成为了最近研究的一大热门[25-27]。据报道, 聚三苯 胺作为锂离子电池正极材料具有高比容量, 高能量密度和 良好的循环稳定性。其所以能表现出良好的电池性能主要 归功于其三苯胺基结构的 $\pi$ 共轭和聚三苯胺的可逆的氧化 还原性质[28]。

有机正极材料的氧化还原电位是由电活性有机基团 所决定的。不同的结构通常显示不同的充放电平台和平均 充放电电压。通常, $\mathrm{p}$-掺杂比 $n$-掺杂的氧化还原电位要高, 例如, 氧氮自由基和聚苯胺导电聚合物的平均充放电电压 为 $3.5 \mathrm{~V}$, 而有机二硫化合物和大多数的共轭羰基化合物的 充放电电压表现出较低的 $3.0 \mathrm{~V}$ 。通过理论计算, 发现官能 团相邻的吸电子基团（例如-Cl,-F,-CN和- $\mathrm{NO}_{2}$ ）或供电子 基团（例如- $\mathrm{OH},-\mathrm{NH}_{2}$ 和 $-\mathrm{OCH}_{3}$ ) 可以分别提高或降低氧化 还原电位 $[29,31]$ 。
在前期相关研究工作中[31], 分别在三苯胺基本单元 上引入具有吸电子和推电子效应的基团, 并聚合导电相应 的聚合物正极材料, 研究发现吸电子基团将提高电极的电 压平台, 且聚三苯胺氧基具有较高的平台和较好的放电比 容量。因此, 在本论文中, 首先将具有吸电子效应的吡啶 基团引入三苯胺中, 制备了 $\mathrm{N}, \mathrm{N}$-二苯基吡啶-4-胺单体 (DPN) 以及对应的均聚物(PDPN)。并考察了聚 $\mathrm{N}, \mathrm{N}-二$ 二苯 基吡啶-4-胺结构与电化学性能之间的关系以及作为正极 材料所表现出的电池性能。

\section{2. 实验部分}

\section{1. 药品与试剂}

二苯胺（99\%），三氯化铁（99\%），N-甲基吡咯烷 酮（NMP， 99.9\%, AR）购自阿拉丁试剂公司; 叔丁醇钾 （98\%），4-澳吡啶盐酸盐(98\%)，2,2'-双-(二苯膦基)-1,1'联荎BINAP $(98 \%)$ 购买自安耐吉化学。醋酸钯 $(47.5 \% \mathrm{Pd})$ 购买自百灵威试剂有限公司。

\section{2. 聚 $\mathrm{N}, \mathrm{N}$-二苯基吡啶-4-胺材料制备}

$\mathrm{N}, \mathrm{N}$-二苯基吡啶-4-胺单体的合成: 在预先经过干燥 处理的 $100 \mathrm{~mL}$ 两口烧瓶中加入 4 - 溴吡啶盐酸盐 $(207 \mathrm{mg}, 1.06 \mathrm{mmol})$, 二 苯胺 $(217 \mathrm{mg}, 1.28 \mathrm{mmol})$, $\mathrm{Pd}(\mathrm{OAc})_{2}(11 \mathrm{mg}, 0.05 \mathrm{mmol}), \operatorname{BINAP}(31 \mathrm{mg}, 0.05 \mathrm{mmol})$ 和 $\mathrm{KOt}-\mathrm{Bu}(321 \mathrm{mg}, 2.85 \mathrm{mmol})$, 通氮气保护, 然后用针筒 注射 $20 \mathrm{~mL}$ 甲苯, 摚拌溶解并升温至 $70^{\circ} \mathrm{C}$ 反应 $1 \mathrm{~h}$ 。反应结 束后, 加水淬灭反应, 反应粗产物用二氯甲烷和水洗涤、 萃取并用无水硫酸镁干燥, 过滤。萃取液采用旋转蒸发仪 去除溶剂, 并用柱色谱将旋蒸后的剩余产物进行分离提纯, 最终得到 $\mathrm{N}, \mathrm{N}-$ 二苯基吡啶-4-胺70mg, 产率 70\%, 产物为 淡黄色固体粉末。

MS(EI):m/z:248.3.1HNMR(500MHz,DMSO) $88.18(\mathrm{dd}, \mathrm{J}$ $=4.8,1.6 \mathrm{~Hz}, 2 \mathrm{H}), 7.47-7.40(\mathrm{~m}, 4 \mathrm{H}), 7.28-7.20(\mathrm{~m}, 6 \mathrm{H}), 6.59(\mathrm{dd}, \mathrm{J}$ $=4.8,1.6 \mathrm{~Hz}, 2 \mathrm{H})$ 。

本章目标化合物DPN单体的合成路线如下图1所示:

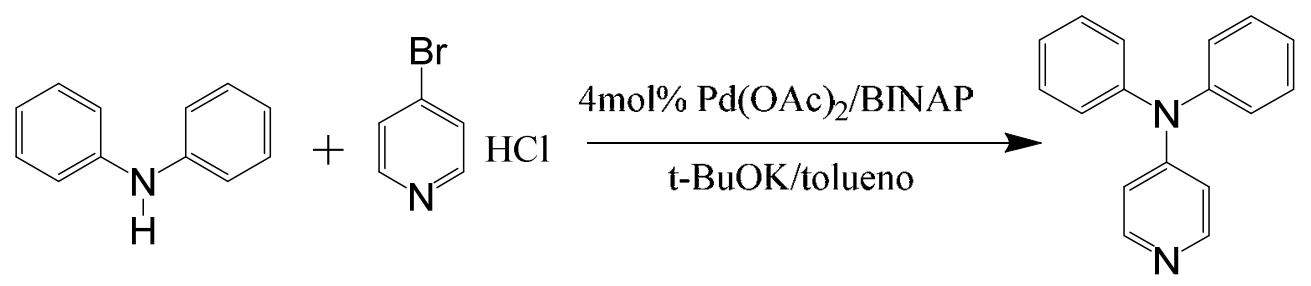

图1 化合物DPN的合成路线。

聚 $\mathrm{N}, \mathrm{N}$-二苯基吡啶-4-胺的合成：聚 $\mathrm{N}, \mathrm{N}$-二苯基吡 啶-4-胺（PDPN）是通过化学氧化法进行制备的, 以无水 三氯化铁为氧化剂。将 $0.5 \mathrm{~g}$ 单体溶于 $30 \mathrm{ml}$ 的氯仿中, 缓慢 加入摩尔量为单体 4.5 倍的氧化剂氯化铁粉末。反应在氮 气保护下进行 $24 \mathrm{~h}$ 。反应结束后, 加入大量的甲醇使产物 沉淀, 然后过滤。用甲醇和水交替洗涤滤饼3次, 将得到 的滤饼在 $60^{\circ} \mathrm{C}$ 下真空干燥 $24 \mathrm{~h}$ 。

\section{3. 表征与测试}

核磁共振波谱（NMR）在瑞士Bruker AVANCE III $500-\mathrm{MHz}$ 型核磁共振谱仪上测试得到。以気代氯仿 $\left(\mathrm{CDCl}_{3}\right)$ 作为溶剂, 四甲基硅烷 (TMS) 作为内标, 浓度控制在 $5 \mathrm{mg} \cdot \mathrm{mL}^{-1}$ 。红外光谱 (FTIR) 数据在美国Nicolet 6700 型傅 立叶变换红外光谱仪采集, 测试波段为 $4000-400 \mathrm{~cm}^{-1}$ 。电 化学测试数据在上海华辰 $\mathrm{CHI} 660 \mathrm{C}$ 型电化学分析仪上采 
集。采用标准三电极槽, 以氧化铟锡导电玻璃（ITO）或 金属 $\mathrm{Pt}$ 作为工作电极, $\mathrm{Pt}$ 片作为对电极, 参比电极为 $\mathrm{Ag} / \mathrm{AgCl}$, 电解液为 $0.1 \mathrm{MTBAP} / \mathrm{CH}_{2} \mathrm{Cl}_{2}$ 。表面形貌观察 (SEM) 采用日本Hitachi S-4800场发射型扫描电子显微镜 进行聚合物表面形貌、颗粒大小和颗粒分布分析, 样品在 观察前进行表面喷金处理。电池性能测试在武汉金诺电子 LAND电池测试系统（CT2001A，5V/10Ma）上采集。电 压范围为 $1.5-4.2 \mathrm{~V}$, 以 $20 \mathrm{~mA} \cdot \mathrm{g}^{-1}$ 的充放电速率进行恒流充 放电性能测试。

\section{4. 电极制备及半电池组装}

电极的制备: 采用涂膜法制备电极, $\mathrm{N}$-甲基吡咯烷酮 (NMP) 作为溶剂, 将原料按照质量比 $\mathrm{m}$ (活性物质) : $\mathrm{m}$ (导电剂乙炔黑) :m (PVDF) $=50: 40: 10$ 的比例混合成正 极浆液, 再将浆液涂在预处理过的铝箔上, 在真空干燥箱 中于 $60^{\circ} \mathrm{C}$ 下干燥 $12 \mathrm{~h}$, 压片 (16Mpa) 后得到正极片。
半电池的组装: 半电池组装是在高纯 $A r$ 保护的手套箱 中完成, 手套箱中的湿度和氧含量保持在 $1 \mathrm{ppm}$ 以下。以 金属锂片为对电极和参比电极, $1 \mathrm{~mol} / \mathrm{LLiPF}_{6} \mathrm{EC}$ : DMC： $\mathrm{EMC}=1: 1: 1(\mathrm{w} / \mathrm{w})$ 溶液为电解液, 聚丙烯微孔膜 (Celgard2300)为隔膜, 组装成CR2032扣式半电池。组装好 的电池进行封口并静置 $12 \mathrm{~h}$, 以便电解液能够在电池两极 之间扩散均匀, 使电解液与电池的两极完全接触。

\section{3. 结果与讨论}

\subsection{FTIR分析}

图2是 $\mathrm{N}, \mathrm{N}$-二苯基吡啶-4-胺（DPN）化合物的单晶 解析图, 从图中可以看出在每一个晶胞中都有八个分子构 成, 且分子排列十分整齐, 两个分子之间通过 $\mathrm{N}-\mathrm{H}$ 氢键连 接, 氢键的长度为 $3.556 \AA$ 。单晶的得出进一步证明了我们 所合成的物质是目标产物。

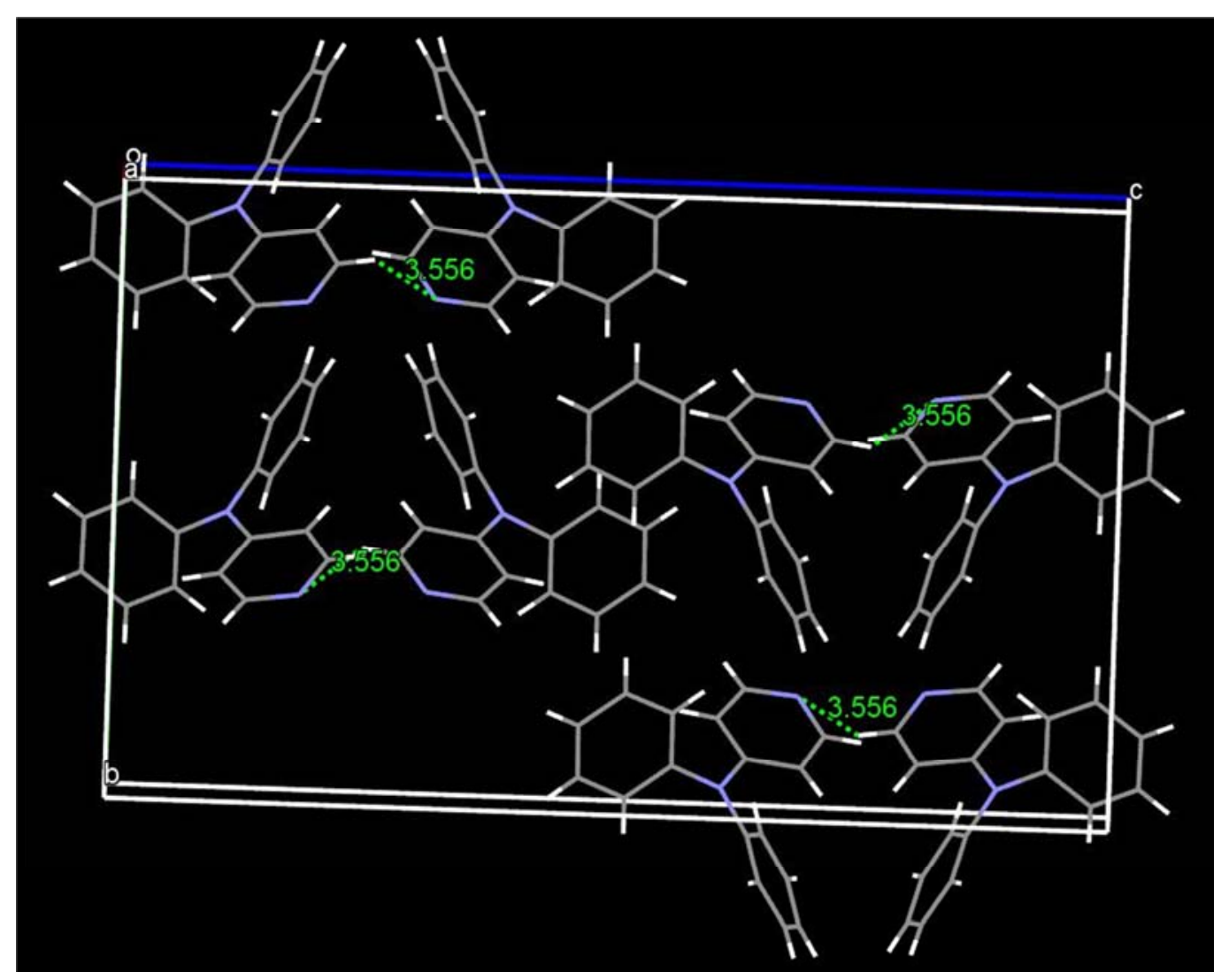

图2 化合物DPN的单晶图。

\subsection{FTIR分析}

如图3所示为两种聚合物PTPAn和PTTPATZ的红外光 谱图。从图中可以看出, 两种聚合物都有三苯胺的特征峰, 位于 $1594 \mathrm{~cm}^{-1}$ 处的吸收峰是苯环上 $\mathrm{C}=\mathrm{C}$ 键的伸缩振动峰, $1490 \mathrm{~cm}^{-1}$ 处出现的吸收峰是苯环上的 C-C 键的伸缩振动峰, $1328 \mathrm{~cm}^{-1}$ 上的吸收峰是由于苯环上的 $\mathrm{C}-\mathrm{H}$ 键的弯曲振动所 引起的, 出现在 $1276 \mathrm{~cm}^{-1}$ 和 $820 \mathrm{~cm}^{-1}$ 处的吸收峰分别是归属
于叔胺 C-N键的伸缩振动和 1,4-双取代苯环上的 C-H键的 面外摇摆振动。而对于PDPN, 出现了吡啶的特征峰, $1384 \mathrm{~cm}^{-1}$ 处出现的吸收峰可以认为是吡啶环 $\mathrm{C}=\mathrm{N}$ 键骨架振 动特征峰, 而 $1639 \mathrm{~cm}^{-1}$ 处出现的是吡啶环上的 $\mathrm{C}=\mathrm{C}$ 键的伸 缩振动峰。所以从PDPN的红外图谱中可以看出, PDPN 的红外结构具备了目标产物的基本基团的红外特征吸收 峰, 可以初步认定该聚合物就是目标产物。 


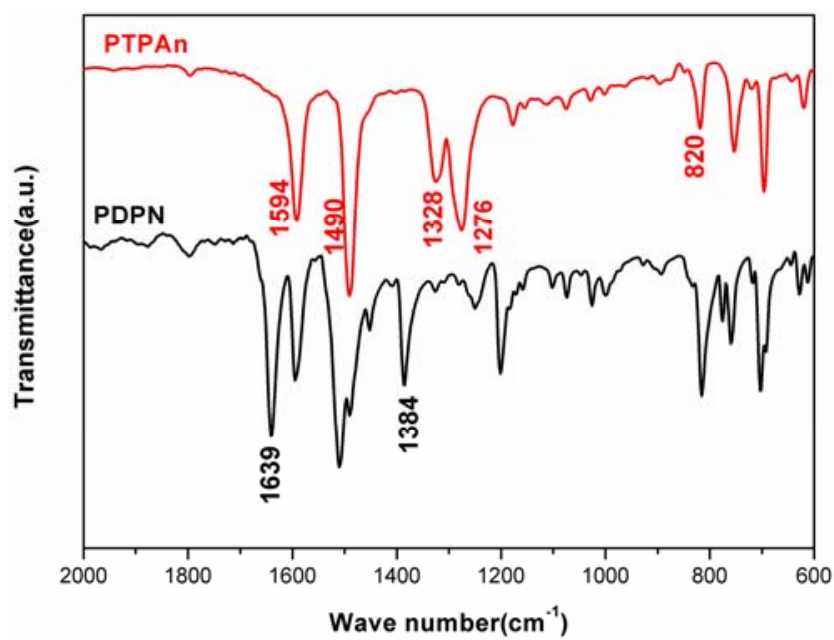

图3 两种聚合物PTPAn和PDPN的红外光谱图。

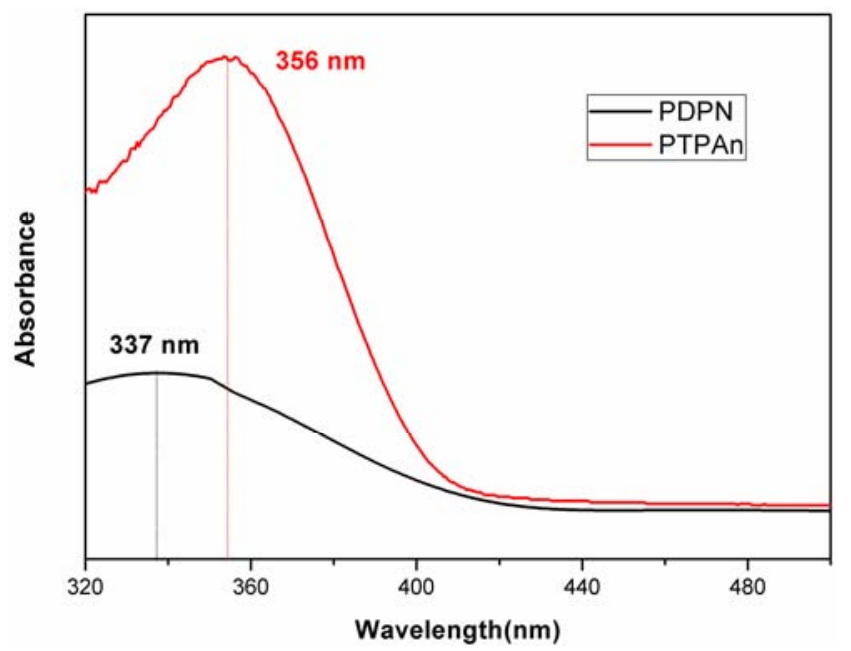

\subsection{UV-Vis分析}

为了进一步确认聚合物的结构, 将聚合物PDPN和 PTPAn分别溶解在DMF溶剂中进行紫外-可见光吸收光 谱测试。从图4中可以看出, PTPAn的紫外吸收峰出现 在 $356 \mathrm{~nm}$ 处强吸收峰, 被归结为三苯胺典型的 $\pi-\pi *$ 电子 跃迁吸收峰。而PDPN在 $337 \mathrm{~nm}$ 处存在强紫外吸收峰, 也被归结为三苯胺单元的 $\pi-\pi$ *电子跃迁吸收峰。相对于 聚三苯胺而言, PDPN聚合物中的三苯胺单元的 $\pi-\pi *$ 电 子跃迁吸收峰有明显的蓝移, 这是因为三苯胺上的一个 苯环被吡啶环取代, 吡啶基团的强吸电子相应导致电子 离域降低, 在UV-Vis 吸收峰上表现为明显的蓝移。研究 结果说明, 吸电子相应基团能明显改变聚三苯胺基础材 料的电子特征, 将对的能级结构和电极材料的电势产生 一定影响。

\subsection{SEM分析}

通过以上测试基本确定了其聚合物的化学结构, 为 了进一步了解聚合物的微观形貌, 将聚合物 PDPN和 PTPAn进行了对比。如图5(a)所示, 在相同的放大倍率下, 聚合物PTPAn颗粒团聚严重且粒子尺寸也较大。相比较 地, 通过在三苯胺结构单元中引入具有吸电子效应的吡 啶环后, 其聚合物颗粒变小且呈现分散状, 形成的粒子 直径大约为 2 微米 (图5(b))。同时也从图中可以看出, 聚三苯胺的团聚体大小不一, 团聚严重, 而PDPN的颗粒 大小均一且分散。这种改善的分散颗粒结构, 导致颗粒 聚集体之间有一定的空隙, 有助于电解液中的离子的自 由进出。有利于作为电极材料过程中与电解质的大面积 接触和粒子在充放电过程中的迁移, 并最终影响带电池 的充放电性能。

图4 PDPN和PTPAn的紫外光谱图(溶于DMF, $10^{-2} \mathrm{~g} \cdot \mathrm{L}^{-1}$ )。

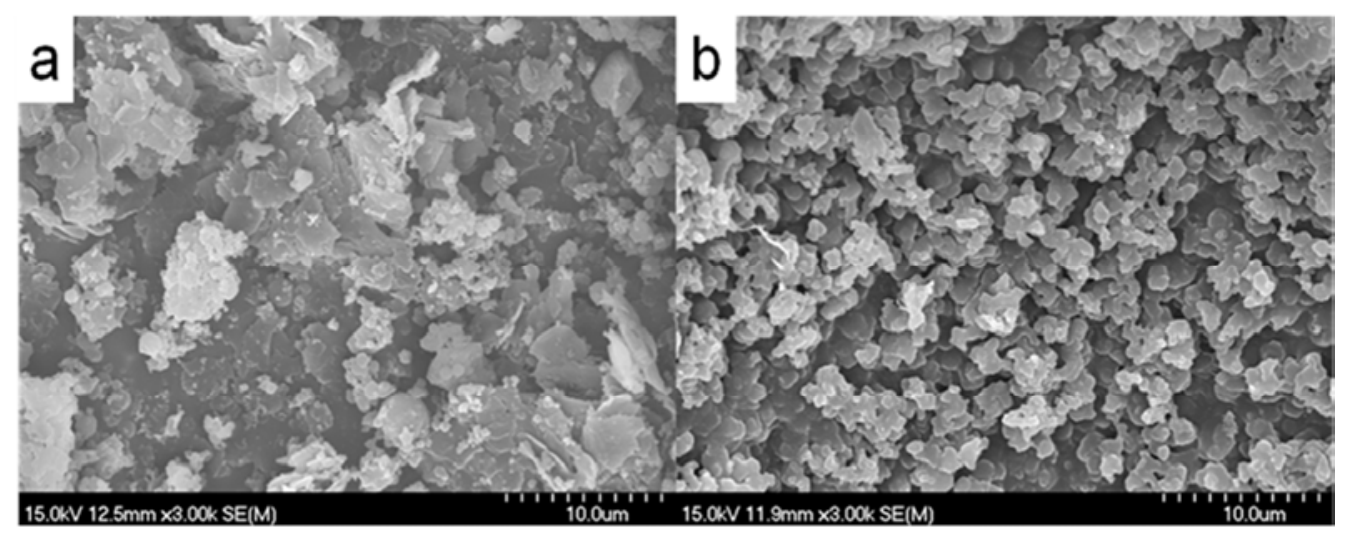

图5 (a)PTPAn和(b)PDPN的扫描电镜图。

\section{5. 循环伏安测试分析}

图6为PDPN的循环伏安曲线, 扫描速率均为 $1 \mathrm{mV} / \mathrm{s}$ 。 电化学测试数据在上海华辰CHI660C型电化学分析仪上 采集。测试采用三电极测试方法, 以氧化铟锡导电玻璃 (ITO) 作为工作电极, $\mathrm{Pt}$ 片作为对电极, 参比电极为 $\mathrm{Ag} / \mathrm{AgCl}$, 电解液为 $0.1 \mathrm{MTBAP} / \mathrm{CH}_{2} \mathrm{Cl}_{2}$ 。从测试结果可以
看出, PDPN作为电极材料具有较为对称的氧化-还原特征 峰, 表明该材料具有一定可逆的氧化-还原电化学反应特 征。从图中也可以看出, PDPN材料在测试范围内分别存 在两对氧化-还原特征峰, 分别位于 $-0.75 /-1.75 \mathrm{~V}$ 和 $-0.46 /-1.25 \mathrm{~V}$ 位置, 表明聚合物在充放电过程中发生两电子 得失过程。并且, 每对氧化峰和还原峰的峰间距较大, 表 明材料充放电过程中存在较大的极化现象。 


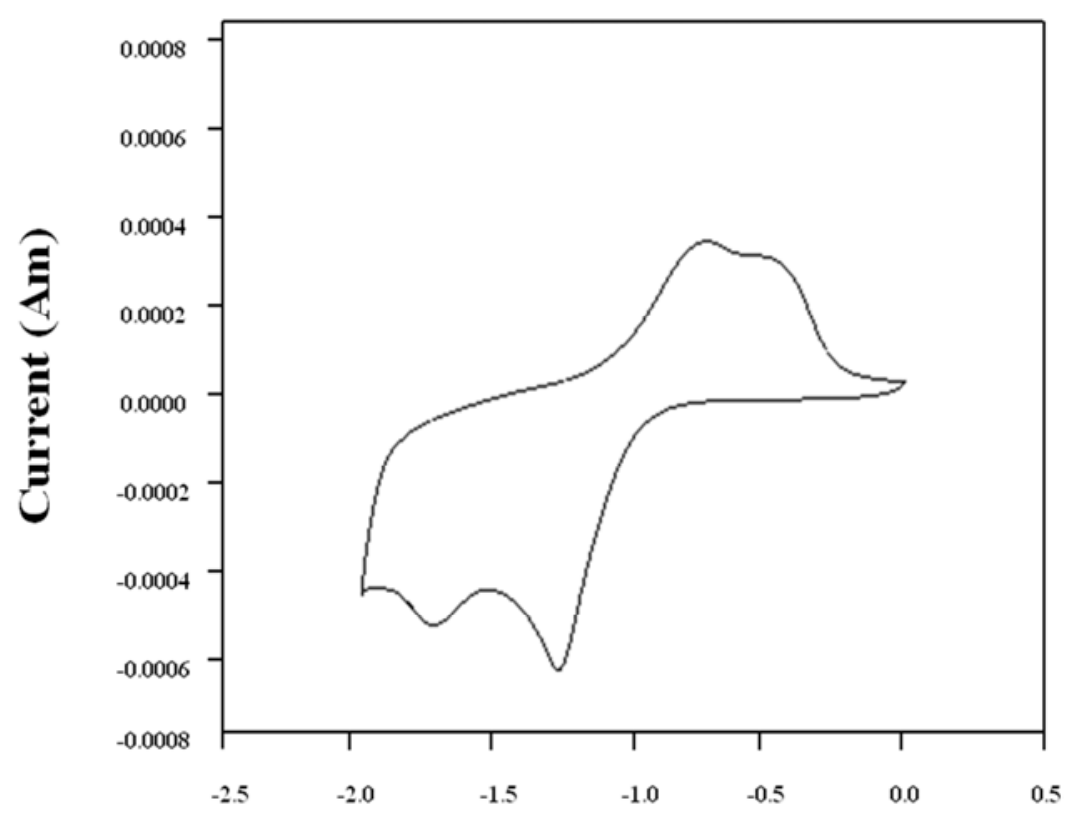

Voltage Vs. Ag/AgCl

图6 PDPN的循环伏安曲线图。

\section{6. 电池性能测试}

为了研究本实验制备的聚 ( $\mathrm{N}, \mathrm{N}-$ 二苯基吡啶-4-胺) （PDPN）的电池性能, 进一步探索引入吸电子基团对电 池电压平台的影响, 将制备的好的聚合物作为活性物质作 为正极材料组装器件, 也就是 CR2032扣式电池, 并进行 充放电性能研究。

图7 为 $\mathrm{PDPN}$ 电极在 $1 \mathrm{MLiPF}_{6} \mathrm{EC} / \mathrm{DMC}(\mathrm{v} / \mathrm{v}, 1: 1)$ 电解质 中, 以 $20 \mathrm{~mA} \cdot \mathrm{g}^{-1}$ 恒定的充放电速率下测试的首次充放电曲
线。由图可知, PDPN电极材料在测试电压范围内首次放 电比容量为 $92.3 \mathrm{mAh} \cdot \mathrm{g}^{-1}$ 。其测试曲线具有明显不同的充放 电曲线特征结构, 在4.1 4.2V和1.5-1.8V电压范围内有两 个明显的电压平台, 其中在 $4.0 \sim 4.2 \mathrm{~V}$ 范围内显现的高电压 平台具有比文献报道的聚三苯胺明显高的放电曲线平台, 可归结为由于吡啶吸电子基团引入所导致的电势升高, 而 1.5-1.8V之间的电压平台是吡啶环上 $\mathrm{N}$ 原子本身得失电子 过程所致。

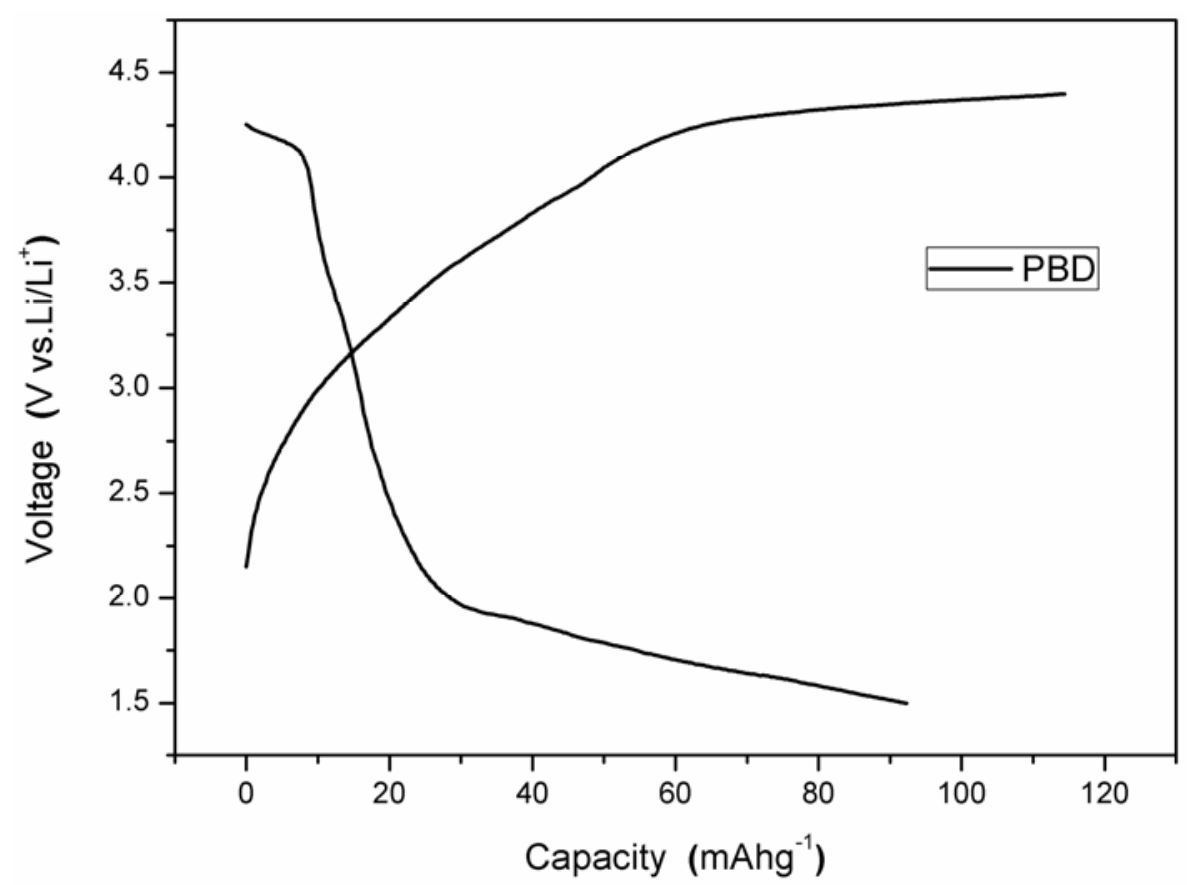

图7 聚合物PDPN在1MLiPF6EC/DMC(v/v,1:1)电解质中 $20 \mathrm{~mA}^{-\mathrm{g}^{-1}}$ 充放电速率下的首次充放电曲线(电压范围: $1.5 \sim 4.3 \mathrm{Vvs} \cdot \mathrm{Li} / \mathrm{Li}^{+}$)。 
图8为聚合物电极 $P D P N$ 在 $1 \mathrm{M} \mathrm{LiPF}_{6} \mathrm{EC} / \mathrm{DMC}(\mathrm{v} / \mathrm{v}, 1: 1)$ 的电解质中, 在1.5-4.2 $\mathrm{V}$ 电压之间, 以 $20 \mathrm{mAg}^{-1}$ 的恒定充放电 速率测试的循环稳定性。如图所示, PDPN电极在循环过程中容量有点波动, 循环性能不稳定, 经过 50 次循环后其实 际容量从刚开始的 $92.3 \mathrm{mAhg}^{-1}$ 衰减到了 $73.2 \mathrm{mAhg}^{-1}$, 大约 $20.7 \%$ 的容量损失。如图所示, 前5圈的循环比较稳定, 到了 第6圈其容量下降，第6圈之后其容量有相对较为稳定。

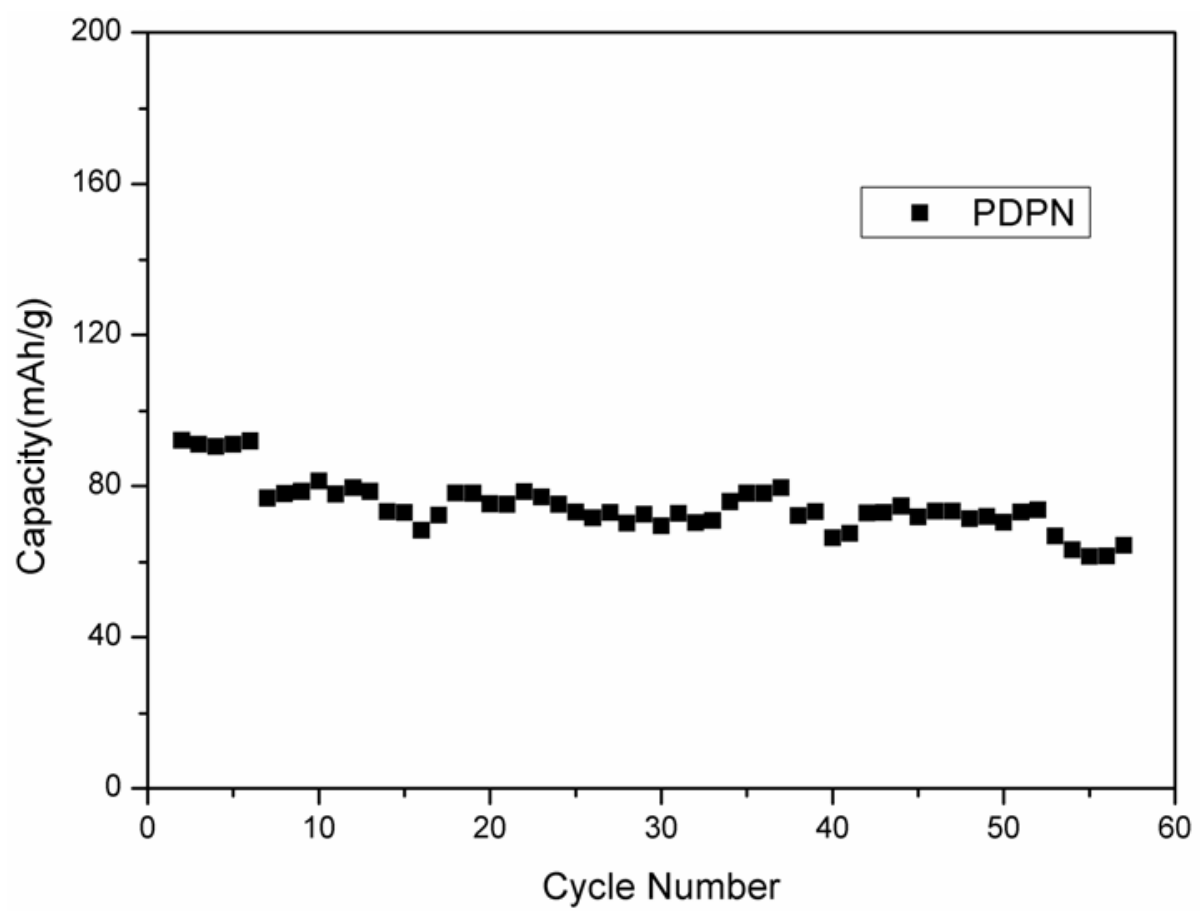

图8 聚合物电极PDPN在 $1 \mathrm{M} \mathrm{LiPF} 6 \mathrm{EC} / \mathrm{DMC}(\mathrm{v} / \mathrm{v}, 1: 1)$ 电解质中 $20 \mathrm{~mA} \cdot \mathrm{g}^{-1}$ 充放电速率下的循环稳定性(电压范围: $\left.1.5 \sim 4.3 \mathrm{Vvs} . \mathrm{Li} / \mathrm{Li}^{+}\right)$。

\section{4. 结论}

本文成功合成 $\mathrm{N}, \mathrm{N}$-二苯基吡啶-4-胺 (DPN) 化合物, 并培养出了其单晶, 然后通过化学氧化法成功制备了其均 聚物-聚（N, N-二苯基吡啶-4-胺）（PDPN）。通过核磁 氢谱和质谱以及单晶的分析确认了单体的化学结构。通过 红外光谱以及紫外光谱测试证实了三种聚合物的结构。电 池性能测试发现, PDPN电极在4.1-4.3V和1.5-1.8V电压范 围内有两个明显的电压平台, 且首次放电比容量为 $92.3 \mathrm{mAh} \cdot \mathrm{g}^{-1}$ 。经过 50 次循环后其实际容量从刚开始的 $92.3 \mathrm{mAhg}^{-1}$ 衰减到了 $73.2 \mathrm{mAhg}^{-1}$, 大约 $20.7 \%$ 的容量损失。

\section{致谢}

本文为国家自然科学基金一般项目《含均三嗪结构聚 三苯胺微介孔有机锂电池正极材料的制备与电化学性能 研究》(51573099); 辽宁省自然科学基金项目《微介孔结 构聚三苯胺衍生物有机锂电池正极材料的研究》 (2015020441)、《聚苯胺/ $\mathrm{Fe}(\mathrm{CN})_{6}{ }^{3-} /$ 石墨烯化碳管杂化锂电 池正极材料的研究》(201602591); 辽宁省高校重点实验室 项目《基于聚三苯胺衍生物/石墨烯复合材料的有机自由 基聚合物锂电池正极材料的基础研究》(LZ2016005); 辽 宁省高等学校创新人才支持计划项目(LR2017034); 辽宁 省教育厅项目《原位自聚合法制备导电高分子修饰的普鲁
士蓝金属配位化合物锂电池正极材料的研究》(LQ2017010) 资助。

\section{参考文献}

[1] Petr Novák, Klaus Müller, K. S. V. Santhanam, Otto Haas, Electrochemically active polymers for rechargeable batteries [J], Chemical Reviews, 1997, 97(1): 207-282.

[2] Koji Nishio, Masahisa Fujimoto, Noriyuki Yoshinaga, Nobuhiro Furukawa. Characteristics of a lithium secondary battery using chemically-synthesized conductive polymers [J], Journal of Power Sources, 1991, 34(2): 153-160.

[3] Vernitskaya, Tat'yana Vladimirovna, Oleg Nikolaevich Efimov, Polypyrrole: a conducting polymer; its synthesis,properties and applications [J], Russian Chemical Reviews, 1997, 66: 443-457.

[4] David MacInnes Jr., Mark A. Druy, Paul J. Nigrey, David P. Nairns, Alan G. MacDiarmid, Alan J. Heeger, Organic batteries:Reversible n-type and p-type electrochemical doping of polyacetylene, $(\mathrm{CH})_{\mathrm{X}}[\mathrm{J}]$, Journal of the Chemical Society, Chemical Communications, 1981: 317-322.

[5] Futoshi Matsumoto, Masahide Ozaki, Yu Inatomi, Scott C. Paulson, Noboru Oyama, Studies on the adsorption behavior of 2,5-Dimercapto-1,3,4-thiadiazole and 2-Mercapto-5-methyl-1,3,4-thiadiazole at gold and copper electrode surfaces [J], Langmuir, 1999, 15: 857-865. 
[6] Katsuhiko Naoi, Kenichi Kawase, Mitsuhiro Mori, Michiko Komiyama, Electrochemistry of poly(2,2'-dithiodianiline): A new class of high energy conducting polymer interconnected with S-S bonds [J], Journal of the Electrochemical Society, 1997, 144(6): L173-L175.

[7] Jinxia Li, Hui Zhan, Lei Zhou, Shiren Deng, Zaoying Li, Yunhong Zhou, Aniline-based polyorganodisulfide redox system of high energy for secondary lithium batteries [J], Electrochemistry Communications, 2004,6(6): 515-519.

[8] Yu Zhi Su, Yong Ping Niu, Yi Zhi Xiao, Min Xiao, Zhao Xi Liang, Ke Cheng Gong, Novel conducting polymer poly[bis(phenylamino)disulfide]: synthesis, characterization and properties[J], Journal of Polymer Science Part A: Polymer Chemistry, 2004, 42(10): 2329-2339.

[9] Haiyan Chen, Michel Armand Dr., Gilles Demailly Prof., Franck Dolhem Dr., Philippe Poizot Dr., Jean-Marie Tarascon Prof, From biomass to a renewable $\mathrm{LixC}_{6} \mathrm{O}_{6}$ organic electrode for sustainable Li-ion batteries [J], ChemSusChem, 2008,1(4): 348-355.

[10] Wesley Walker, Sylvie Grugeon, Olivier Mentre, Stéphane Laruelle, Jean Marie Tarascon, Fred Wudl, Ethoxycarbonyl-based organic electrode for Li-batteries [J], Journal of the American Chemical Society, 2010, 132(18): 6517-6523.

[11] Haiyan Chen, Michel Armand, Matthieu Courty, Meng Jiang, Clare P. Grey, Franck Dolhem, Jean Marie Tarascon, Philippe Poizot, Lithium salt of tetrahydroxybenzoquinone: toward the development of a sustainable Li-ion battery [J], Journal of the American Chemical Society, 2009, 131(25): 8984-8988.

[12] Daniel Häringera, Petr Nováka, Otto Haasa, Benoît Pirob and Minh - Chau Pham, Poly(5-amino-1,4-naphthoquinone), a novel lithium-inserting electroactive polymer with high specific charge [J], Journal of The Electrochemical Society, 1999, 146: 2393-2397.

[13] Miao Ken Hung, Yu Hsuan Wang, Chun-Hao Lin, Hsiao Chien Lin and Jyh Tsung Lee, Synthesis and electrochemical behavior of nitroxide polymer brush thin-film electrodes for organic radical batteries [J], Journal of Materials Chemistry, 2012, 22: 1570-1577.

[14] Soo Gil Park, Koichi Aoki, Koichi Tokuda, Hiroaki Matsuda, Electrochemical behavior of nitroxy radical monolayers prepared on tin oxide electrodes by the langmuir-blodgett method [J], Journal of Electroanalytical Chemistry and Interfacial Electrochemistry,1985, 195(1): 157-163.

[15] Takeo Suga, Shuhei Sugita, Hiroki Ohshiro, Kenichi Oyaizu, Hiroyuki Nishide, p- and n-type bipolar redox-active radical polymer: toward totally organic polymer-based rechargeable devices with variable configuration $[\mathrm{J}]$, Advanced Materials, 2011, 23(6): 751-754.

[16] Masahiro Suguro, Shigeyuki Iwasa, Yuki Kusachi, Yukiko Morioka, Kentaro Nakahara, Cationic polymerization of poly(vinylether) bearing a TEMPO radical: A new cathode-active mMaterial for organic radical batteries [J], Macromolecular Rapid Communications, 2007, 28(18): 1929-1933.

[17] Ullrich Mitschke, Peter Bäuerle, The electroluminescence of organic materials $[\mathrm{J}]$, Journal of Materials Chemistry, 2000, 10(7): 1471-1507.
[18] Yunchuan Li, Xiang Long Li, Xinyi Cai, Dongcheng Chen, Xin Liu, Gaozhan Xie, Zhiheng Wang, Yuan Chun Wu, Chang Cheng Lo, A. Lien, Junbiao Peng, Yong Cao, Shi Jian $\mathrm{Su}$, Deep blue fluorophores incorporating sulfone-locked triphenylamine: the key for highly efficient fluorescencephosphorescence hybrid white OLEDs with simplified structure [J], Journal of Materials Chemistry C, 2015, 3(27): 6986-6996.

[19] Gopal Singh, Vandana Bhalla, Manoj Kumar, Carbazole end-capped and triphenylamine-centered starburst derivative for hole-transport in electroluminescent devices [J], Optical Materials, 2015, 46: 82-87.

[20] Antonio Abate, Sanghyun Paek, Fabrizio Giordano, Juan Pablo Correa-Baena, Michael Saliba, Peng Gao, Taisuke Matsui, Jaejung Ko, Shaik M. Zakeeruddin, Klaus H. Dahmen, Anders Hagfeldt, Michael Grätze, Silolothiophene-linked triphenylamines as stable hole transporting materials for high efficiency perovskite solar cells [J], Energy \& Environmental Science, 2015, 8(10): 2946-2953.

[21] Shifan Wang, Jie Yang, Zhiguo Zhang, Yuanyuan Hu, Xudong Cao, Hai Li, Youtian Tao, Yongfang Li, Wei Huang, A new V-shaped triphenylamine/diketopyrrolopyrrole containing donor material for small molecule organic solar cells [J], RSC Advances, 2015, 5(83): 68192-68199.

[22] Andreas Petr, Carita Kvarnström, Lothar Dunsch, Ari Ivaska, Electrochemical synthesis of electroactive polytriphenylamine [J], Synthetic metals, 2000, 108(3): 245-247.

[23] Sylwia Golba, Oliwia Starczewska, Krzysztof Idzik, Electrochemical and spectrophotometric properties of polymers based on derivatives of di-and triphenylamines as promising materials for electronic applications [J], Designed Monomers and Polymers, 2015, 18(8): 770-779.

[24] Sandra Pluczyk, Pawel Zassowski, Cassandre Quinton, Pierre Audebert, Valérie Alain-Rizzo, Mieczyslaw Lapkowski, Unusual electrochemical properties of the electropolymerized thin layer based on a s-tetrazine-triphenylamine monomer [J], The Journal of Physical Chemistry C, 2016.120(8):4382-4391.

[25] Wei Nia, Jianli Chenga, Xiaodong Lia, Guifang Gua, Ling Huanga, Qun Guan, Demao Yuana, Bin Wang, Polymeric cathode materials of electroactive conducting poly (triphenylamine) with optimized structures for potential organic pseudo-capacitors with higher cut-off voltage and energy density [J], RSC Advances, 2015, 5(12): 9221-9227.

[26] Mark E. Roberts, David R. Wheeler, Bonnie B. McKenzie,Bruce C. Bunker, High specific capacitance conducting polymer supercapacitor electrodes based on poly (tris (thiophenylphenyl) amine) [J], Journal of Materials Chemistry, 2009, 19(38): 6977-6979.

[27] Jeremy T. Kearns, Mark E. Roberts, Synthesis of high-charge capacity triarylamine-thiophene redox electrodes using electrochemical copolymerization [J], Journal of Materials Chemistry, 2012, 22(48): 25447-25452.

[28] J.K. Feng, Y.L. Cao, X.P. Ai, H.X. Yang, Polytriphenylamine: A high power and high capacity cathode material for rechargeable lithium batteries [J], Journal of Power Sources, 2008, 177(1): 199-204. 
[29] Mansoor Namazian, Hora A. Almodarresieh, Computational electrochemistry: aqueous two-electron reduction potentials for substituted quinones [J], Journal of Molecular Structure: THEOCHEM, 2004, 686(1): 97-102.

[30] Kamal Alizadeha, Mojtaba Shamsipur, Calculation of the two-step reduction potentials of some quinones in acetonitrile $[\mathrm{J}]$, Journal of Molecular Structure: THEOCHEM, 2008, 862(1): 39-43.
[31] Chang Su, Xiaogang Zhu, Lihuang Xu, Ningning Zhou, Huihui He, Cheng Zhang, Organic polytriphenylamine derivative-based cathode with tailored voltage and its electrochemical performances [J], Electrochimica Acta, 2016, 196: 440-449. 